\title{
Uso de endoprótesis fenestrada para la reparación de aneurismas aórticos complejos. Reporte de dos casos*
}

\author{
Drs. MICHEL BERGOEING R. ${ }^{1}$, RENATO MERTENS M. ${ }^{1}$, FRANCISCO VALDÉS E. ${ }^{1}$, \\ LEOPOLDO MARINÉ M. ${ }^{1}$, ALBRECHT KRÄMER SCH. ${ }^{1}$, EU JEANNETTE VERGARA M. ${ }^{1}$ \\ 1 Departamento de Cirugía Vascular y Endovascular, División de Cirugía, Facultad de Medicina, \\ Pontificia Universidad Católica de Chile. \\ Santiago, Chile.
}

\begin{abstract}
Fenestrated endografts for the treatment of complex aortic aneurysms. Report of two cases

In the last decade endovascular repair of infrarenal aortic aneurysms (EVAR) has become increasingly popular. However, until recently patients with juxtarenal abdominal aortic aneurysms (JAAA) or with thoracoabdominal aortic aneurysms (TAA) were not candidates for EVAR due to the lack of an adequate landing zone to deploy the endograft. Because of considerable morbidity and mortality that traditional open surgery of these aneurysms entail, new endografts with fenestrations and branches have been developed to treat these patients. We present our initial experience with two cases, both male with coronary artery disease considered high-risk for traditional open repair. The first patient has a $4.1 \mathrm{~cm}$ sacular JAAA; it is repaired with a fenestrated endograft with branches for both renal arteries (RA), superior mesenteric artery (SMA) and a scallop for the celiac trunk (CT). The second patient has a $5.9 \mathrm{~cm}$ TAA with a previous aorto bifemoral bypass; because the CT is chronically occluded it is repaired with a fenestrated endograft with branches for both RA and SMA. In both patients post operative course was uneventful. Follow-up at 11 months and 30 days respectively, show adequate exclusion of the aneurysm with patency of all revascularized vessels. This new therapeutic procedure allows treatment of high-risk patients with complex aortic aneurysms in whom conventional repair entails a prohibitive surgical risk.
\end{abstract}

Key words: Thoracoabdominal aortic aneurysm, abdominal aortic aneurysm, stents, aorta.

\section{Resumen}

La reparación endovascular de un aneurisma aórtico abdominal infrarrenal (EVAR) se ha popularizado en la última década. Sin embargo, hasta ahora los pacientes con aneurisma aórtico abdominal yuxtarrenal (AAAY) o aneurisma aórtico tóracoabdominal (AATA) no eran candidatos a EVAR por ausencia de una zona sana donde apoyar la endoprótesis tubular. La reparación convencional se asocia a una morbimortalidad con-

*Recibido el 7 de diciembre de 2010 y aceptado para publicación el 30 de diciembre de 2010.

Correspondencia: Dr. Michel Bergoeing R.

Marcoleta 367 Piso 8. Fax: +56 (2) 354-3268

mbergoei@med.puc.cl 
siderable, por lo que se han desarrollado endoprótesis capaces de acomodar ramas de la aorta que permiten tratar estos aneurismas en forma mínimamente invasiva. Presentamos la experiencia inicial de dos casos, ambos de sexo masculino y portadores de enfermedad coronaria considerados de alto riesgo para cirugía abierta. El primero, portador de un AAAY sacular de 4,1 cm de diámetro; se repara mediante el uso de endoprótesis fenestrada con ramas a ambas arterias renales (AR), arteria mesentérica superior (AMS) y una escotadura para el tronco celíaco (TC). El otro, portador de AATA de 5,9 cm de diámetro, un puente aorto bifemoral previo y TC crónicamente ocluido; se repara con endoprótesis fenestrada con ramas para las AR y AMS. Ambos pacientes presentaron una evolución post operatoria favorable. El seguimiento a 11 meses para el primero y 30 días para el segundo demuestra exclusión del aneurisma y permeabilidad de todas las arterias revascularizadas. Este nuevo procedimiento terapéutico abre la posibilidad de tratar pacientes de alto riesgo, portadores de aneurismas aórticos complejos, para los que una alternativa convencional implica un alto riesgo quirúrgico.

Palabras clave: Aneurisma aórtico toracoabdominal, aneurisma aórtico abdominal, prótesis, aorta.

\section{Introducción}

Desde su creación por Parodi hace 20 años $^{1}$, la reparación endovascular (EVAR) ha pasado a ser la técnica más popular en países desarrollados para tratar el aneurisma aórtico abdominal infrarrenal (AAA), si la anatomía es adecuada. Estudios contemporáneos demuestran resultados comparables con la cirugía convencional ${ }^{1-3}$.

Sin embargo, los pacientes que no presentan condiciones anatómicas para recibir una endoprótesis, por ausencia de una zona normal de aorta donde apoyar y sellar el dispositivo, se enfrentan a una situación completamente distinta. Los aneurismas de aorta abdominal yuxtarrenales (AAAY), que nacen inmediatamente por debajo de las arterias renales, o los aneurismas tóraco-abdominales (AATA), que comprometen la aorta torácica descendente y simultáneamente las ramas viscerales de la aorta, presentan en manos expertas cifras de mortalidad para su reparación abierta que van de $5 \%$ a $9 \%{ }^{4-7}$ y riesgo de paraplejia de $2,7 \%$ a $33 \%$ en el caso de un AATA ${ }^{8,9}$. En busca de mejorar estos resultados, se han desarrollado otras alternativas terapéuticas.

Una alternativa para resolver por vía endovascular este complejo problema clínico, es utilizar una endoprótesis que por diseño permita la revascularización simultánea de las ramas viscerales. Estos dispositivos han mostrado resultados clínicos iniciales prometedores ${ }^{10}$. A continuación presentamos nuestra experiencia inicial en 2 pacientes con el uso de estos dispositivos.

\section{Caso 1}

Hombre de 75 años de edad, diabético tipo II, en insuficiencia renal crónica con creatininemia de 2,59 mg/dL e hipertensión arterial crónica (HTA), es hospitalizado en otro centro por dolor lumbar intenso, fiebre y artritis séptica de tobillo, expuesta a través de una úlcera isquémica crónica a nivel del maléolo peroneo. Como hallazgo se evidencia un AAAY de tipo sacular de 4,1 cm de diámetro (Figura 1A). Se traslada a nuestro centro para continuar su tratamiento. El paciente evoluciona con infarto agudo del miocardio (IAM); se realiza coronariografía que no demuestra lesiones revascularizables, por lo que se trata en forma médica satisfactoriamente. La tomografía por emisión de positrones descarta compromiso séptico de la aorta y demuestra una espondilodiscitis T5-T6, iniciando antibioticoterapia. Se resuelve la isquemia de su extremidad inferior mediante revascularización con puente distal cicatrizando su úlcera y resolviendo la artritis séptica. Debido a la forma sacular del aneurisma, se plantea su reparación quirúrgica diferida.

Resuelto su cuadro séptico, reingresa para tratamiento de su AAAY. Considerando su alto riesgo quirúrgico, se elige tratar en forma mínimamente invasiva, mediante endoprótesis ramificada. Por vía femoral se implanta una endoprótesis con fenestraciones para ambas arterias renales (AR) y la arteria mesentérica superior (AMS), además de una escotadura para acomodar el tronco celíaco (TC) (COOK Medical Inc., EEUU); para las ramas renales se utilizaron stents cubiertos V12 (Atrium Medical Corporation, EEUU) y para la AMS un stent autoexpansible SMART Control (Cordis Corporation, EEUU).

En el postoperatorio no presenta complicaciones, ni deterioro de su reducida función renal, siendo dado de alta al $6^{\circ}$ día post operatorio. El control alejado con imágenes se realiza con ecografía dúplex para evitar la administración de contraste, mostrando a los 4 y 11 meses permeabilidad de las reconstrucciones y exclusión del aneurisma (Figura 1B).

\section{Caso 2}

Hombre de 63 años de edad, cardiópata coronario con antecedentes de cirugía de revascularización miocárdica a los 51 años; HTA y diabético tipo II. Es intervenido por un AAA a los 56 años en otro centro mediante puente aorto bifemoral. En seguimiento mediante imágenes se detecta un AATA de $5,9 \mathrm{~cm}$ de diámetro que se inicia a nivel del cuerpo vertebral T9 y termina inmediatamente por encima 
del puente aórtico previo; presenta adicionalmente oclusión asintomática del TC (Figura 1C). Estudio de perfusión miocárdica (cintigrama ${ }^{99 \mathrm{~m}}$ Tc sestamibi) demuestra secuela de infarto antiguo e isquemia residual mayor al $10 \%$ de la pared lateral y una fracción de eyección del ventrículo izquierdo de 35\%. Por esto se considera de alto riesgo para cirugía convencional, planteando reparación endovascular.

Considerando la extensión del AATA, el tratamiento implica excluir múltiples arterias intercostales y por su cirugía aórtica previa presenta un alto riesgo de isquemia medular ${ }^{5,7}$. Por este motivo se emplea drenaje de líquido céfalo raquídeo para disminuir la presión intratecal y optimizar la perfusión medular. Por vía femoral se despliega endoprótesis con fenestraciones para ambas AR y AMS (COOK Medical Inc., EEUU). Se utilizaron stents cubiertos V12 (Atrium Medical Corporation, EEUU) para las tres ramas viscerales y la AMS se complementó con un stent autoexpansible SMART Control (Cordis Corporation, EEUU). Se completó la reparación hacia el tórax con una endoprótesis aórtica TX2 (COOK Medical Inc., EEUU) y hacia distal la endoprótesis se ancló adecuadamente en la prótesis aorto bifemoral antigua de dacrón.

Cursa el postoperatorio sin incidentes y es dado de alta al $6^{\circ}$ día. La tomografía axial computada a los 30 días demuestra exclusión total del aneurisma, con perfusión adecuada de los vasos revascularizados (Figura 1D).

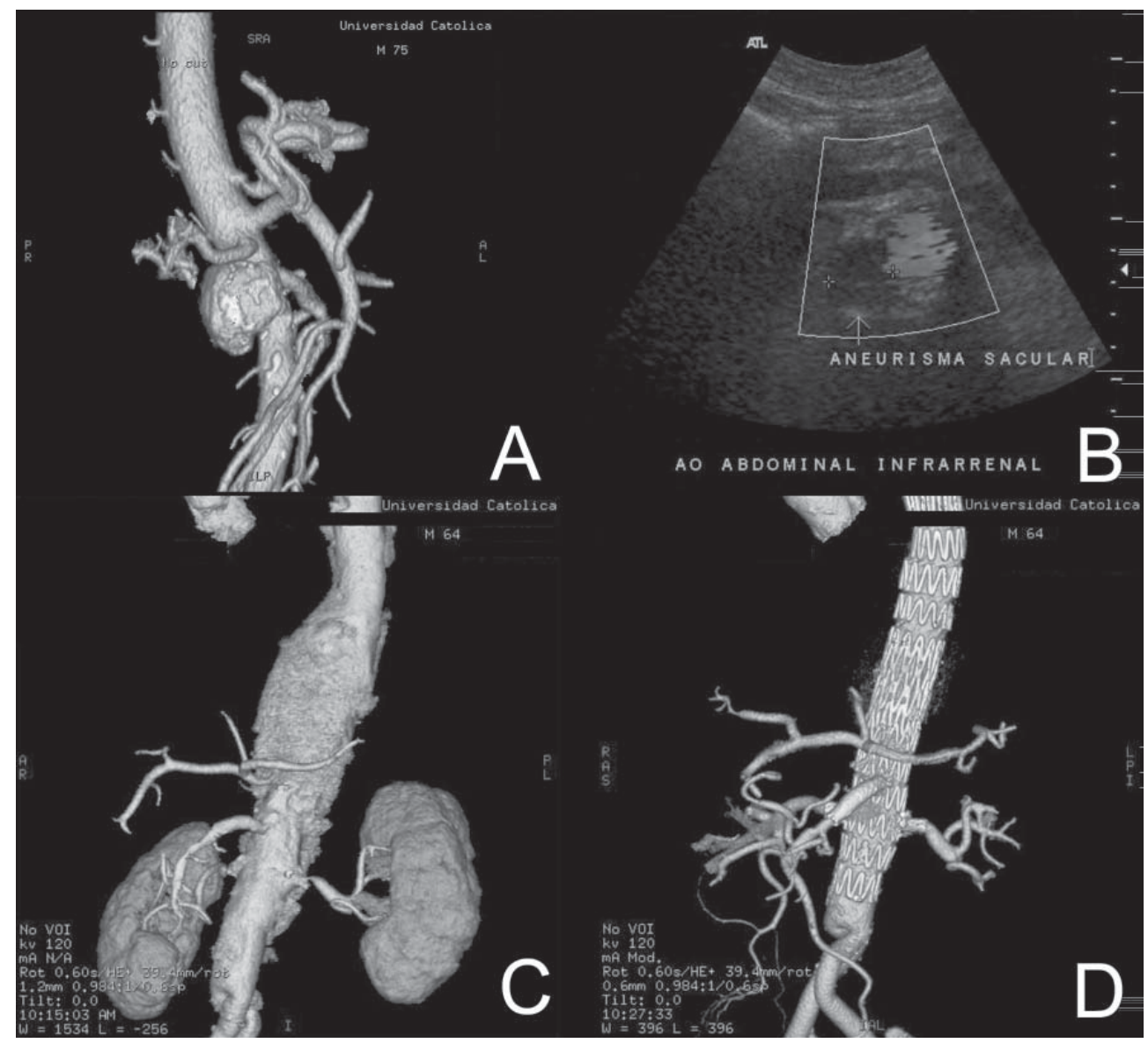

Figura 1. (A) Caso 1, reconstrucción tridimensional de tomografía computada que muestra aneurisma yuxtarrenal sacular, vista lateral derecha. (B) Caso 1, ecografía dúplex control que demuestra corte transversal a nivel de la aorta y el aneurisma sacular. La ecografía demuestra flujo por la endoprótesis y ausencia de flujo en el aneurisma (flecha). (C) Caso 2, reconstrucción tridimensional de tomografía computada preoperatoria que demuestra la extensión del aneurisma tóraco abdominal. El tronco celíaco está ocluido y se llena por ramas colaterales. (D) Caso 2, reconstrucción tridimensional de tomografía computada que demuestra la reparación del aneurisma con la endoprótesis fenestrada, se aprecian los stents a ambas arterias renales y la arteria mesentérica superior. En el extremo inferior se aprecia la prótesis aórtica bifurcada antigua. 


\section{Discusión}

El tratamiento endovascular del aneurisma se basa en la exclusión de éste mediante una endoprótesis que sella cualquier filtración a nivel de los sitios de anclaje proximal y distal a la dilatación. El uso de esta técnica en presencia de ramas relevantes emergiendo del aneurisma a tratar, implicaría su obstrucción, provocando isquemia secundaria. En ese sentido, los AAAY y AATA han presentado hasta ahora una barrera a esta técnica, pues el uso de endoprótesis convencionales produciría isquemia visceral y muerte.

Una alternativa es la realización de cirugía híbrida: convencional y endovascular. En un primer tiempo se realiza una revascularización convencional extra anatómica de los troncos viscerales, seguida del implante de una endoprótesis que excluye al aneurisma ${ }^{11}$. Sin embargo, la mortalidad asociada a esta técnica ha sido más elevada de lo esperado, con cifras que varían entre un $13 \%$ a $23 \%{ }^{12,13}$.

Diversos autores han desarrollado una solución endovascular para estos pacientes ${ }^{14-16}$. Todos se han basado en el uso de una prótesis modular que debe ser ensamblada in situ. Consiste en un cuerpo principal con portales laterales, uno para cada arteria visceral que se desea preservar. Los portales pueden ser agujeros (fenestraciones) o ramas propiamente tales. Las fenestraciones pueden corresponder a escotaduras en el borde superior o inferior de la prótesis, o bien a agujeros. Los agujeros se conectan a las arterias viscerales mediante el uso de stents cubiertos, impermeables a la sangre, que se despliegan en forma separada. A este componente aórtico visceral, se le agregan componentes proximales o distales según la extensión del aneurisma a reparar (Figura 2).

Si bien la posibilidad de resolver estos aneurismas complejos por vía endovascular es muy atractiva, el uso de prótesis fenestradas es complejo. El diseño de la prótesis requiere una meticulosa medición tridimensional del aneurisma a partir de una tomografía computada preoperatoria de alta resolución. Las fenestraciones o ramas deben alinearse perfectamente con las arterias viscerales para permitir su canulación y posterior despliegue del stent cubierto. Esto requiere que cada prótesis sea diseñada y construida a la medida del paciente, lo que significa una demora prolongada y riesgo de ruptura del aneurisma durante la espera. Se encuentra en desarrollo prótesis con fenestraciones para las AR y una escotadura para la AMS y TC que permitirían tratar entre $70 \%$ a $85 \%$ de pacientes con aneurismas pararrenales con una prótesis estándar ${ }^{17}$.

El procedimiento es prolongado, técnicamente demandante y requiere experiencia en cirugía en- dovascular mayor. El riesgo de exposición a radiación para el paciente y el personal médico debe ser considerado $^{18}$. La aplicación de este tratamiento a pacientes pequeños, en especial mujeres, aumenta la complejidad dado el alto diámetro de los sistemas introductores (20-24 Fr), pudiendo ser necesario el acceso retroperitoneal directo a las arterias iliacas. El éxito técnico (revascularización de las ramas viscerales) varía entre $85 \%$ a $100 \%$ según la serie y la mortalidad a 30 días entre $1 \%$ a $8,5 \%$ para AAAY, siendo un poco mayor para AATA, entre 6,3\% a $9,1 \%{ }^{10,15,19}$. Sin embargo, hay que tomar en cuenta que estas series incluyen pacientes de alto riesgo, rechazados para cirugía convencional. Por último, dependiendo de la extensión de la reparación, persiste riesgo de paraplejia de 4,3\% en promedio, ciertamente inferior a la cirugía abierta ${ }^{14,20}$. El seguimiento de por vida es mandatorio, pues existe el riesgo de falla del dispositivo, al igual que en otras reparaciones endovasculares. En especial la oclusión de ramas viscerales, que en su mayoría se asocia a fractura del stent o la separación de componentes con pérdida de sello y presurización del aneurisma previamente excluido ${ }^{20}$.

Si bien series recientes comparan favorablemente la reparación endovascular de estos aneurismas complejos con la reparación abierta tradicional ${ }^{14,15}$, aún no hay información disponible del seguimiento a largo plazo. Aunque es posible que al igual que EVAR esta técnica se haga más popular, por ahora está reservada sólo para pacientes de alto riesgo quirúrgico, adecuadamente informados y tratados por un equipo con experiencia en cirugía endovascular compleja.

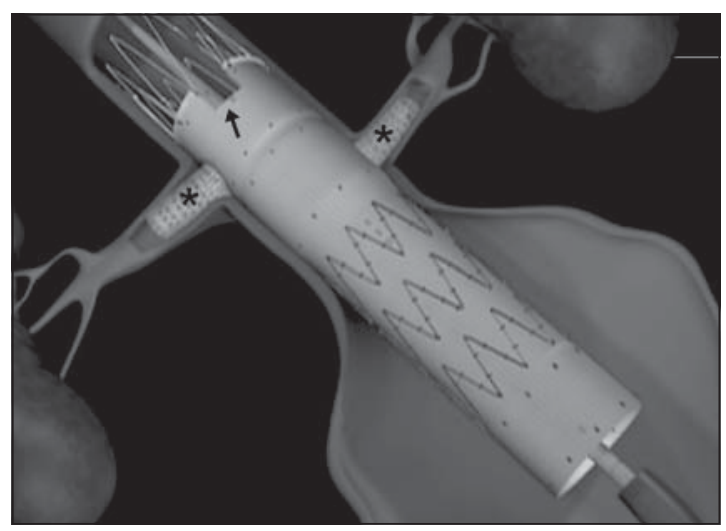

Figura 2. Componente principal de endoprótesis fenestrada. Los asteríscos $(*)$ muestran los stents renales. La flecha señala la escotadura para acomodar la arteria mesentérica superior. 


\section{Referencias}

1. Greenhalgh RM, Brown LC, Powell JT, Thompson SG, Epstein D, Sculpher MJ. Endovascular versus open repair of abdominal aortic aneurysm. N Engl J Med. 2010; 362:1863-71.

2. De Bruin JL, Baas AF, Buth J, Prinssen M, Verhoeven EL, Cuypers PW, et al. Long-term outcome of open or endovascular repair of abdominal aortic aneurysm. $\mathrm{N}$ Engl J Med. 2010;362:1881-9.

3. Valdés F, Mertens R, Kramer A, Bergoeing M, Marine L, Canessa R, y cols. Reparación endovascular de aneurisma de aorta abdominal. Resultados en 80 pacientes consecutivos. Rev Med Chile 2006;134:1265-74.

4. Crawford ES, Crawford JL, Safi HJ, Coselli JS, Hess KR, Brooks B, et al. Thoracoabdominal aortic aneurysms: preoperative and intraoperative factors determining immediate and long-term results of operations in 605 patients. J Vasc Surg. 1986;3:389-404.

5. Svensson LG, Crawford ES, Hess KR, Coselli J S, Safi HJ. Experience with 1509 patients undergoing thoracoabdominal aortic operations. J Vasc Surg. 1993; 17:357-68; discussion 368-70.

6. Sarac TP, Clair DG, Hertzer NR, Greenberg RK, Krajewski LP, O’Hara PJ, et al. Contemporary results of juxtarenal aneurysm repair. J Vasc Surg. 2002;36:110411.

7. Coselli JS, Bozinovski J, LeMaire SA. Open Surgical Repair of 2286 Thoracoabdominal Aortic Aneurysms. The Annals of Thoracic Surgery 2007;83:S862-4.

8. Cinà CS, Abouzahr L, Arena GO, Laganà A, Devereaux PJ, Farrokhyar F. Cerebrospinal fluid drainage to prevent paraplegia during thoracic and thoracoabdominal aortic aneurysm surgery: a systematic review and metaanalysis. Journal of Vascular Surgery 2004;40:36-44.

9. Khan SN, Stansby G. Cerebrospinal fluid drainage for thoracic and thoracoabdominal aortic aneurysm surgery. Cochrane Database Syst Rev. 2004:CD003635.

10. Greenberg RK, Lytle B. Endovascular Repair of Thoracoabdominal Aneurysms. Circulation. 2008;117:228896.

11. Mertens MR, Valdés EF, Kramer Sch A, Marine ML,
Bergoeing RM, Sagues CR, y cols. Revascularización extra anatómica y tratamiento endovascular para la reparación de un aneurisma toracoabdominal. Informe de cuatro casos. Rev Med Chile 2007;135:153-9.

12. Black SA, Wolfe JH, Clark M, Hamady M, Cheshire NJ, Jenkins MP. Complex thoracoabdominal aortic aneurysms: endovascular exclusion with visceral revascularization. J Vasc Surg. 2006;43:1081-9; discussion 1089.

13. Chiesa R, Tshomba Y, Melissano G, Marone EM, Bertoglio L, Setacci F, et al. Hybrid approach to thoracoabdominal aortic aneurysms in patients with prior aortic surgery. J Vasc Surg. 2007;45:1128-35.

14. Greenberg RK, Lu Q, Roselli EE, Svensson LG, Moon MC, Hernández AV, et al. Contemporary Analysis of Descending Thoracic and Thoracoabdominal Aneurysm Repair: A Comparison of Endovascular and Open Techniques. Circulation 2008;118:808-17.

15. Reilly LM, Chuter TA. Endovascular repair of thoracoabdominal aneurysms: design options, device construct, patient selection and complications. J Cardiovasc Surg (Torino) 2009;50:447-60.

16. Verhoeven EL, Tielliu IF, Ferreira M, Zipfel B, Adam D J. Thoraco-abdominal aortic aneurysm branched repair. J Cardiovasc Surg (Torino) 2010;51:149-55.

17. Greenberg RK, Qureshi M. Fenestrated and branched devices in the pipeline. Journal of Vascular Surgery 2010;52:15S-21S.

18. Panuccio G, Greenberg RK, Wunderle K, Mastracci T, Eagleton MJ, Davros W. A Comparison of Indirect Radiation Dose Estimates With Directly Measured Radiation Dose for Patients and Operators During Complex Endovascular Procedures. Journal of Vascular Surgery 2010;51:6S-6S.

19. Monahan TS, Schneider DB. Fenestrated and branched stent grafts for repair of complex aortic aneurysms. Semin Vasc Surg. 2009;22:132-9.

20. Dowdall JF, Greenberg RK, West K, MoonM, Lu Q, Francis C, et al. Separation of components in fenestrated and branched endovascular grafting-branch protection or a potentially new mode of failure? Eur J Vasc Endovasc Surg 2008;36:2-9. 